\title{
Peer discrimination of obese children. Research conducted based on a programme for dealing with aggression organised for children attending elementary school
}

\author{
Małgorzata Obara-Gołębiowska \\ Department of Psychology of Development and Education, University of Warmia and Mazury, Olsztyn, Poland \\ Department of Obesity Management, United Municipal Hospital, Olsztyn, Poland
}

\begin{abstract}
Introduction: Obese children are victims of weight-based discrimination and stigmatisation. The objective of the study was to analyse the expansion of the phenomenon of peer discrimination against obese children in a group of children at early school age.

Material and methods: The study was performed in an elementary school among four classes of the third grade where psychoeducational classes were conducted regarding ways of dealing with violence at school. There were 98 children aged 9-10 years and 4 teachers participating in the study. The study involved an analysis of the content of the performed drawings, and presentation of interviews with teachers regarding statements made by children during the discussion. The study focused on the phenomenon of peer violence towards obese children.

Results: In 47 (47.96\%) prepared drawings, aggression associated with obesity was observed. Interviews showed that the children had a negative stereotype of obesity and they frequently experienced or observed cases of teasing obese peers.

Conclusions: Weight-based discrimination and violence towards obese children are frequently observed or experienced among children aged 9-10 years. Social interventions are required aimed at reducing the level of bias towards obese children, as well as psychopedagogical assistance in this group of children.
\end{abstract}

Key words: obesity, children, peer discrimination, stigmatisation, violence.

\section{Introduction}

According to the available epidemiological studies, $16.4 \%$ of Polish teenagers and children are overweight or obese [1]. In addition to physical consequences of excessive weight, obesity also results in a number of psychosocial problems. Obese children are victims of weight-based discrimination and stigmatisation. They are dissatisfied with their own appearance, and their self-esteem is generally lower than that of other children [2]. The consequences of stigmatisation have a physiological dimension. It turns out that this type of traumatic experience has a psychosomatic impact. It directly strengthens impaired glucose tolerance, insulin resistance, hypertension, and dyslipidaemia [3]. The sources of discrimination of obese children are diverse. Children are sometimes discriminated at home by their siblings and parents, and at school by peers [4]. The first publication concerning discrimination of obese children by peers date back to the 1960s. One of the classic studies concerning the issue was performed by Richardson et al. [5]. Students aged 10-11 years were given a task of ordering six photographs of other students, thus indicating which children they would potentially make friends with. Four photographs presented children with disabilities and with a deformed face, the fifth photograph presented an overweight child, and the sixth showed a child with no excess weight or any forms of disability. It turned out the obese child was assessed the lowest as a candidate for a potential friend.

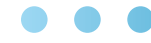

$\bullet \bullet \bullet$ 
Negative attitudes towards overweight and obese peers appear already in the third year of age. For example, one of the studies showed that three-year-olds ascribed "fat" children with a number of negative characteristics such as: mean, stupid, loud, ugly, lazy, sad, and unpopular [6]. Due to comparisons with others, already several-year-olds are aware of being fat. Moreover, they develop a negative stereotype of a "fatso" which, combined with their own obesity, leads to a decrease in self-esteem. Among older children, the tendency for negative perception of peers with excessive weight increases. This is confirmed by results of research on children aged 4-11 years. They used descriptions of obese persons such as: ugly, selfish, lazy, stupid, liar, dirty, unfair, and devious. Moreover, persons with excessive weight were also discriminated as partners of team games, and especially as leaders of such groups [7]. Girls are particularly sensitive to negative comments regarding their appearance, and they believe they will be more popular if they lose weight. Moreover, due to their different appearance or weaker physical condition, obese children are often mocked, rejected by their class, and adopt the role of the clown or scapegoat of the class more frequently [7]. Stigmatisation leads to the exclusion of obese children from the community of the class and school, considerably affecting their self-esteem and the general image of their future social functioning. It was found that approximately $36 \%$ of obese boys and $34 \%$ of obese girls become victims of physical and particularly psychological violence. Interviews conducted with 50 obese girls showed that $95 \%$ of them were subject to mean comments of other people, social rejection, and general unfair treatment caused by excessive body weight [7]. Stigmatisation, negatively influencing self-esteem, also substantially contributes to more frequent occurrence of depression among obese children and teenagers. Results of research by Eisenberg et al. revealed that mocking excessive body weight considerably increas-

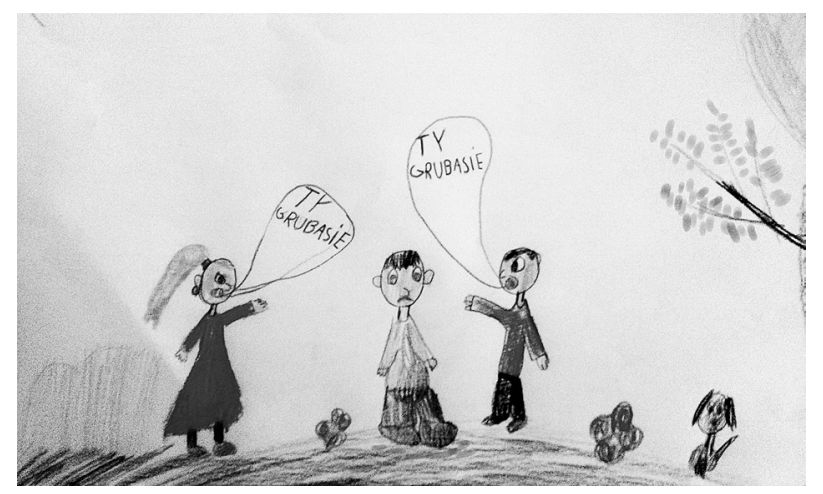

Figure 1. Drawing showing aggression related to obesity (made by 9-year-old girl) es the probability of occurrence of depression in obese children, irrespective of their gender or race [8]. In his research, Neumark-Sztainer obtained results suggesting that $51 \%$ of girls and $13 \%$ of boys mocked by peers due to their excessive body weight had suicidal thoughts, in comparison to $25 \%$ of girls and $4 \%$ of boys who were not subject to this type of mockery $[9,10]$.

The aim of the study was to analyse the expansion of the phenomenon of peer discrimination against obese children based on the analysis of drawings and interviews in a group of children at an early school age.

\section{Material and methods}

The study was performed in an elementary school near Olsztyn among four classes of third grade where psychoeducational classes were conducted regarding ways of dealing with violence at school. The classes were attended by 98 children aged $9-10$ years ( 52 girls and 46 boys) as well as four teachers. The participation in classes was voluntary for children. In the scope of the aforementioned classes, the children were given the task of drawing a situation of peer violence in which they participated or which they observed or experienced. The task was supposed to be made within 30 minutes. Then, a discussion was conducted in the classes between children and teachers concerning the illustrated examples. The study involved the analysis of the content of the performed drawings, and presentation of structured interviews with teachers regarding statements made by children during the discussion (What was the number of children experiencing peer aggression in relation to weight? What types of peer aggression in relation to weight were observed? What examples of peer aggression in relation to weight were observed?). The study focused on the phenomenon of peer violence towards obese children.

\section{Results}

The analysis covered 98 drawings illustrating various cases of peer aggression. There were five competent judges (one psychologist and four pedagogues) assessing pictures according to the type of aggression (dimension: relevant or irrelevant for the study). In 47 (47.96\%) prepared drawings, among others aggression associated with obesity was observed (relevant for the study). Also there were different types of peer aggression (irrelevant for the study) observed in 51 (52.04\%) prepared drawings. The works (relevant for the study) presented children who insulted and mocked their peers by using insulting expressions related to obesity, e.g. "You fatso", "You are so fat"; "Fatso, fatso", "Disgusting fatso". One of the drawings showing aggression related to obesity is presented below based on the consent of the child and the parents (Fig. 1). 
Moreover, interviews conducted with teachers showed that the discussion included statements suggesting that children have a negative stereotype of obesity. Calling someone "fat" was one of the frequent verbal acute forms of demeaning another person irrespective of the actual body weight of the person subject to the insult. Moreover, children mentioned frequently observed cases of teasing obese peers, particularly in cases of verbal and relational abuse. Examples were provided of bullying, harassing, and social isolation of obese children, e.g. "My friend complained that two girls from another class shouted to her in the locker room: 'Move over, fatso!", "No one wants to be in a team with fat children, because they ruin everything", "When I want to tease my cousin, I tell her she is fat, and then she gets really angry", "I hears boys arguing on a break, and one called the other a stupid fatso. The other was a bit fat", "My friend said she wouldn't invite her neighbour to her birthday, because she does not like her, and because the neighbour is a fatso, and she would eat everything and nothing would be left for the others".

\section{Discussion}

Despite significant attention to the medical impacts of obesity, the negative outcomes that obese children experience as a result of stigma, bias, and discrimination are often ignored. Research strongly suggests, however, that bullying and harassment of obese children are common among peers, especially in a school setting [7-10]. The present study also revealed that the problem of stigmatisation and discrimination of obesity among children attending elementary school is quite common. Approximately half of the drawings illustrating examples of peer aggression showed aggression towards an obese child. Also in the discussion between children and teachers regarding peer violence, the issue of bullying and harassment of obese children appeared very frequently. Overweight children need and deserve better protection against harassers, because a hostile environment at school causes a discriminatory impact on their educational opportunities [8]. They should receive protection from harassment and discrimination to ensure that their educational opportunities do not suffer. Reducing stigmatization towards obese children should be part of a comprehensive public health approach to the obesity epidemic. Teasing is associated with lower levels of self-esteem, higher levels of depression, and eating disorder symptoms in overweight children. This can make it difficult for obese children to implement guidelines necessary for weight reduction, such as healthy eating habits, or participating in physical activity [11]. A factor protecting obese children from weight-related discrimination and its psychophysical consequences may be psychoeducational activities aimed at supporting the obese child, and teaching the child efficient strategies of dealing with peer victimization. Programmes assisting overweight children in feeling more comfortable in their school setting may increase their psychophysical comfort and willingness to engage in healthy weight control behaviours.

According to research, pedagogical personnel play one of the most important roles in activities for the reduction of anti-fat bias in schools [12]. Teachers have the potential to help create a positive school environment for obese youth. They can play a critical role in implementing interventions to decrease victimization of obese children and youth. The objective can be met, among other ways, by presenting obese individuals in a positive light during lessons [13], and treating weight-related stigmatization with the same degree of intolerance as other forms of discrimination [9]. Instituting and enforcement of anti-weight teasing rules in school is also an important issue [12]. It seems, however, that the greatest challenge in counteracting weight-based discrimination and victimization of obese children among peers is the impact on the entire society in the scope of attitudes towards obese individuals. This is especially important taking into account that children irrespective of their age, gender, and weight demonstrate negative stereotypes for not only obese peers but also obese adults [14]. The common social idealisation of a slim figure generates opinions according to which being obese is undesirable. This directly contributes to sustaining weight-related stigmatization. According to Davison et al. [15], the social message of the negativity of being obese can cause an association in children that obese people are bad people. It was found that messages conveying negative stereotypes of obese individuals, and idealization of thin people, mostly come from mass media [10]. Due to this, it seems that a mass media campaign directed at the development of tolerant attitudes towards somatic differences such as being obese could be effective in minimizing the degree to which children internalise the thin ideal [15]. Victimization of obese peers is often related to internal bias against an obese status. Therefore coping with weight bias at a societal level may have a crucial impact on the peer discrimination of obese children.

\section{Conclusions}

The research conducted during psychoeducational classes regarding peer violence among 98 children aged 9-10 years showed frequent cases of observed or experienced violence towards obese children. Social interventions are required aimed at reducing the level of bias towards obese children, as well as psychopedagogical assistance in this group of children.

\section{Disclosure}

Author reports no conflict of interest. 


\section{References}

1. Kulaga Z, Litwin M, Tkaczyk M, et al. The height-, weight-, and BMI-for-age of Polish school-aged children and adolescents relative to international and local growth references. BMC Public Health 2010; 10: 109-120.

2. Mirza NM, Davis D, Yanovski JA. Body satisfaction, self-esteem and overweight among inner-city Hispanic children and adolescents. J Adolescent Health 2005; 36: 16-267.

3. Daniels SR. The consequences of childhood overweight and obesity. Future Child 2006; 16: 47-67.

4. McCormack LA, Laska MN, Gray C, et al. Weight-related teasing in a racially diverse sample of sixth-grade children. J Am Diet Assoc 2011; 111: 431-436.

5. Richardson SA, Goodman N, Hastorf AH, et al. Cultural uniformity in reaction to physical disabilities. Am Sociol Rev 1961; 26: 241-247.

6. Gray WN, Kahhan NA, Janicke DM. Peer victimization and pediatric obesity: A review of the literature. Psychol Schools 2009; 46: 720-727.

7. Griffiths LJ, Wolke D, Page AS, et al. Obesity and bullying. Different effects for boys and girls. Arch Dis Child 2006; 91: 121-125.

8. Eisenberg ME, Neumark-Sztainer D, Story M. Associations of weight-based teasing and emotional well-being among adolescents. Arch Pediat Adol Med 2003; 157: 733-738.

9. Neumark-Sztainer D. Weight teasing among adolescents. Correlations with weight status and disordered eating behaviors. Int J Obesity 2002; 26: 123-131.

10. Neumark-Sztainer D. Can we simultaneously work toward the prevention of obesity and eating disorders in children and adolescents? Int J Eat Disorder 2005; 38: 220-227.

11. Storch EA, Milsom VA, Debraganza N, et al. Peer victimization, psychosocial adjustment, and physical activity in overweight and atrisk-for-overweight youth. J Pediatr Psychol 2007; 32: 80-89.

12. Eisenberg ME, Neumark-Sztainer D, Haines J, et al. Weight-based teasing and emotional well-being in adolescents: Longitudinal findings from Project EAT. J Adolescent Health 2006; 38: 675-683.

13. Cramer P, Steinwert T. Thin is good, fat is bad: how early does it begin? J Appl Dev Psychol 1998; 19: 429-451.

14. Tiggemann M, Anesbury T. Negative stereotyping of obesity in children: The role of controllability beliefs. J Appl Soc Psychol 2000; 30: 1977-1993.

15. Davison KK, Birch LL. Predictors of fat stereotypes among 9-year-old girls and their parents. Obes Res 2004; 12: 86-94. 